\title{
Effect of maraviroc on the \\ Correspondence
} pharmacokinetics of

\section{midazolam, lamivudine/}

\section{zidovudine, and} ethinyloestradiol/ levonorgestrel in healthy volunteers

Caroline E. Ridgway, MSc, Pfizer Global Research and Development, Sandwich, UK.

Tel: +44 1304648636

Fax: +44 1304652108

E-mail: caroline.ridgway@pfizer.com

Keywords

bioavailability, drug interactions, exposure, maraviroc, pharmacokinetics

Received

5 November 2007

Accepted

11 January 2008

Samantha Abel, Deborah Russell, Lyndsey A. Whitlock, Caroline E. Ridgway \& Gary J. Muirhead

Pfizer Global R\&D, Sandwich, UK

\section{AIMS}

To assess the effect of maraviroc on the pharmacokinetics of midazolam, a sensitive probe CYP3A4 substrate; lamivudine/zidovudine, a combination of nucleoside reverse transcriptase inhibitors (NRTIs); and ethinyloestradiol/levonorgestrel, a combination oral contraceptive.

\section{METHODS}

Three randomized, double-blind, placebo-controlled studies were conducted in healthy subjects to assess the effect of maraviroc on pharmacokinetics of other drugs. Two, two-period crossover studies were conducted to assess (i) the effect of steady-state maraviroc (300 mg b.i.d.) on pharmacokinetics of midazolam; and (ii) the effect of steady-state maraviroc ( 300 mg b.i.d.) on the pharmacokinetics of lamivudine/zidovudine. A third two-way crossover study was conducted to evaluate the effect of steady-state maraviroc (100 mg b.i.d.) on the pharmacokinetics of $30 \mu \mathrm{g}$ ethinyloestradiol/150 $\mu \mathrm{g}$ levonorgestrel (Microgynon ${ }^{\circledR}$ ).

\section{RESULTS}

The geometric mean ratios for $C_{\max }$ and AUC for each of the compounds tested in the presence and absence of maraviroc were between $92 \%$ and $121 \%$. There were no notable differences in $T_{\max }, t_{1 / 2}$ or $\mathrm{CL}_{R}$ (where measured) for any of the compounds.

\section{CONCLUSIONS}

Maraviroc had no clinically relevant effects on the pharmacokinetics of the CYP3A4 substrate midazolam, the NRTIs zidovudine/lamivudine, or the oral contraceptive steroids ethinyloestradiol and levonorgestrel.

\section{Introduction}

An essential part of evaluating the dosing recommendations for new drugs is the investigation of potential drug interactions. Concurrent administration of agents that affect pharmacokinetics can result in either an increase or decrease in drug exposure, and can significantly alter the safety and/or efficacy profile of either drug [1-3]. Knowledge of major metabolic and clearance mecha- nisms for relevant drugs, and any potential each drug may have for interfering with these mechanisms, can enlighten strategies for complex pharmacotherapy programmes.

Human immunodeficiency virus (HIV)-infected patients are frequently prescribed highly active antiretroviral therapy (HAART) regimens which typically consist of at least three different drugs [4]. These patients also have a high prevalence of comorbid conditions that may require 
other medications [5]. Therefore, examination of drug interactions is a requisite step in the development of new HIV drugs, to determine their suitability for introduction into existing treatment regimens.

Maraviroc is a CCR5 antagonist recently approved for the treatment of HIV-1 infection. Maraviroc is extensively metabolized in man. In vitro data suggest that cytochrome P450 3A4 (CYP3A4) is the primary enzyme involved, with negligible metabolic activity by the other major CYP enzymes [6]. Findings from clinical studies suggest that maraviroc [7] is unlikely to inhibit any of these isozymes at clinically relevant concentrations. Maraviroc also undergoes some renal elimination in man, although its contribution is a relatively small component $(23 \%)$ of total clearance [8].

CYP3A4 is responsible for the metabolism of a large proportion of all known therapeutic drugs [9], many of which are likely to be co-administered with maraviroc. In addition, many of the commonly prescribed HIV therapies are renally cleared. As maraviroc is a substrate for CYP3A4 and renal clearance mechanisms, it is necessary to examine the potential for maraviroc to affect the pharmacokinetics of co-administered agents that are cleared by these routes.

The studies described here were conducted to investigate any effect of maraviroc on probe substrates or commonly co-administered drugs with a range of different clearance mechanisms. Midazolam, a benzodiazepine widely used as a sedative-hypnotic agent in surgical procedures, is metabolized by CYP3A4 and has been adopted as a reliable probe for investigating CYP3A4 drug interactions [10]. A Phase I study was conducted to determine the effect of maraviroc on the pharmacokinetics of a single oral dose of midazolam to ascertain possible induction or inhibition of CYP3A4 by maraviroc in healthy volunteers (study 1).

Nucleoside reverse transcriptase inhibitors (NRTIs) form the backbone of HAART in the treatment of HIV infection. CombivirTM, the combination of the two NRTIs, lamivudine (LMV) and zidovudine (ZDV), is a common component of HAART. LMV is extensively renally eliminated [11], whereas ZDV is primarily eliminated by hepatic non-CYP metabolism [12], with some renal clearance. Renal interactions have previously been noted for LMV and ZDV $[13,14]$. As a small proportion of maraviroc is also renally cleared, and active transport processes are believed to be involved, a study was conducted to investigate the effect of maraviroc on the steady-state pharmacokinetics and renal clearance $\left(C_{R}\right)$ of $L M V$ and $Z D V$ in healthy volunteers (study 2).

Many HIV-infected patients are women of childbearing age who take oral contraceptives. Hence, there was an obvious need to assess the potential of maraviroc to alter the pharmacokinetics of oral contraceptives before progression to advanced-phase patient studies. The common oestrogenic and progestogenic components of oral contraceptives are metabolized by a number of different enzymes, including CYPs, UDP-glucuronosyltransferases, and sulphotransferases [15-17]. The third study summarized here describes the effects of maraviroc on the pharmacokinetics of the oral contraceptive components, ethinyloestradiol (EE) and levonorgestrel (LN) in healthy women (study 3).

\section{Methods}

In studies 1 (midazolam) and 2 (LMV/ZDV), subjects were healthy men and women aged $18-45$ years with body weight between 60 and $100 \mathrm{~kg}$ (men) or 50 and $100 \mathrm{~kg}$ (women) and a body mass index of $18-28 \mathrm{~kg} \mathrm{~m}^{-2}$. In both studies, enrolled women were either surgically sterilized (tubal ligation or hysterectomy), postmenopausal, or were successfully practising contraception during the 3 months prior to entering the study. Both postmenopausal women and potentially fertile women had a negative pregnancy test at screening and immediately before each study period. In study 3 (oral contraceptives), only surgically sterilized women aged 18-45 years with body weight between 50 and $100 \mathrm{~kg}$ and a body mass index of $18-30 \mathrm{~kg} \mathrm{~m}^{-2}$ were included. Subjects were excluded from all studies if they had any evidence of clinically significant disease, positive serology for HIV-1, hepatitis C or hepatitis $B$ virus, laboratory abnormalities, cardiac dysrhythmias, or clinically significant allergies, especially drug hypersensitivity. All studies were conducted in compliance with the principles set forth in the Declaration of Helsinki (1989) and were approved by an independent ethics committee. All subjects provided written informed consent before participation.

\section{Study designs}

All three studies were double-blind, placebo-controlled, two-period crossover studies in healthy subjects. A schematic of study designs for all three studies is presented in Figure 1 .In study 1 ,each treatment period lasted 7 days and the treatment periods were separated by a wash-out period of at least 7 days. Subjects were randomized to receive either maraviroc $300 \mathrm{mg}$ b.i.d. or matching placebo on days 1-6 of each treatment period with a final dose on the morning of day 7 . All subjects received a single oral tablet dose of $7.5 \mathrm{mg}$ of midazolam on the morning of day 7 of both treatment periods. The 300-mg b.i.d. maraviroc dose was chosen for this study because this was considered the highest anticipated clinical dose. The 7.5-mg midazolam dose was chosen because this is the lowest strength tablet dose available, and it is also half of the normal clinical dose, which would avoid excessive sedation during the study, even if significant metabolic inhibition occurred.

In study 2, each treatment period lasted 7 days, and the two treatment periods were separated by a wash-out period of at least 7 days. In each treatment period, subjects received one Combivir ${ }^{\mathrm{TM}}$ tablet (GlaxoSmithKline; $150 \mathrm{mg}$ 

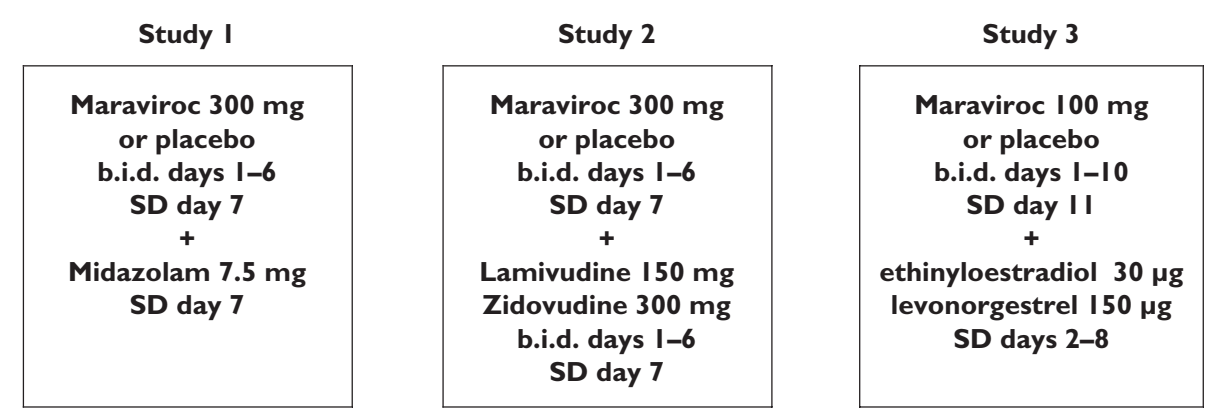

\section{Figure 1}

Study schematics for the three Phase I pharmacokinetics studies. The treatment sequence may have begun with maraviroc or placebo depending on randomization schedules. Days represent days of treatment per treatment period. Following treatment period 1, there was a wash-out period of at least 7 days and then subjects crossed over to the other treatment. SD, single dose

LMV, $300 \mathrm{mg}$ ZDV) b.i.d. and either maraviroc $300 \mathrm{mg}$ b.i.d. or matching placebo b.i.d. on days $1-6$, with a final dose on the morning of day 7. The dose of ZDV/LMV chosen was that used in clinical practice at the time the study was conducted.

In study 3, each treatment period lasted 11 days and the two treatment periods were separated by a wash-out period of at least 7 days. Subjects took either maraviroc $100 \mathrm{mg}$ b.i.d. or matching placebo b.i.d. on days 1-10, with a final dose on the morning of day 11 . Subjects also took Microgynon ${ }^{\circledR} 30$ (Schering Health Care; $30 \mu \mathrm{g} \mathrm{EE}, 150 \mu \mathrm{g}$ $\mathrm{LN}$ ) concomitant with the morning dose of either maraviroc or placebo on days $2-8$. The dose of maraviroc was chosen as it was safe and well tolerated in healthy male volunteers [7] and so was considered appropriate for the first dose in a female population. It was also expected to be within the clinically efficacious dose range at the time the study was conducted. The dose of Microgynon ${ }^{\circledR} 30$ was the standard prescribed dose for effective oral contraception.

\section{Pharmacokinetic assessments}

Blood and urine samples For all studies, blood samples were collected into beadless, heparinized tubes; plasma was isolated by centrifugation and stored at $-20^{\circ} \mathrm{C}$ within $1 \mathrm{~h}$ of collection. Urine samples were collected and stored at $-20^{\circ} \mathrm{C}$ until assayed. For midazolam pharmacokinetics in study 1 , blood samples were collected predose and at intervals up to $24 \mathrm{~h}$ postdose on day 7 . For determination of plasma maraviroc trough concentrations in study 1 , blood samples were collected from subjects predose on days 1, 4 and 7. For determination of plasma pharmacokinetics of LMV and ZDV in study 2, blood samples (one for LMV and one for ZDV) were collected predose on days 1,2 , 4,6 and 7 and at intervals up to $12 \mathrm{~h}$ postdose on day 7 . On day 7 , urine samples were also collected from immediately predose until $12 \mathrm{~h}$ postdose. For assessment of EE and LN plasma pharmacokinetics in study 3, blood samples were collected predose and at intervals up to $24 \mathrm{~h}$ postdose on day 8 . For assessment of plasma maraviroc pharmacokinet- ics in study 3, blood samples were collected predose and then at intervals up to $12 \mathrm{~h}$ postdose on day 1 , predose on days $2,4,6,8,10$ and 11 and at intervals up to $72 \mathrm{~h}$ postdose on day 11 .

Assay details Plasma samples in study 1 were assayed for midazolam by Cephac Europe (Poitiers, France) using a validated method employing solid-phase extraction followed by liquid chromatography and mass spectrometric detection. The overall method imprecision values for analysis of plasma quality control (QC) samples were $13.22 \%, 8.30 \%$ and $13.15 \%$ at target midazolam concentrations of $0.3,50$ and $80 \mathrm{ng} \mathrm{ml}^{-1}$, respectively. The mean inaccuracy of the assay ranged from $1.3 \%$ to $3.4 \%$ over the QC concentration range. The calibration range was $0.1-100 \mathrm{ng} \mathrm{ml}^{-1}$. Plasma samples were analysed for maraviroc by Maxxam Analytics, Inc. (Mississauga, Ontario, Canada) using a validated method employing solid-phase extraction followed by reverse-phase liquid chromatography and mass spectrometric detection. Mean inaccuracy of the assay was $2.0 \%$, $-6.2 \%$ and $-5.0 \%$ for maraviroc at target concentrations of 1,90 and $180 \mathrm{ng} \mathrm{ml}^{-1}$, respectively, and the calibration range was $0.5-200 \mathrm{ng} \mathrm{ml}^{-1}$.

Both plasma and urine samples in study 2 were analysed at Advion Biosciences (Ithaca, NY, USA) for LMV and ZDV using a validated method employing solid-phase extraction followed by liquid chromatography and mass spectrometric detection. The overall method imprecision values for the analysis of plasma QC samples were $6.9 \%$, $4.8 \%$ and $6.3 \%$ for LMV and $8.0 \%, 3.0 \%$ and $5.0 \%$ for ZDV at concentrations of 6,500 and $800 \mathrm{ng} \mathrm{ml}^{-1}$, respectively. The mean inaccuracy of the assay ranged from $-12.1 \%$ to $-1.2 \%$ for LMV and $-5.0 \%$ to $0.8 \%$ for ZDV over the calibration range. The overall method imprecision values for the analysis of urine samples were $3.8 \%, 3.8 \%$ and $3.8 \%$ for LMV and $2.9 \%, 2.1 \%$ and $1.3 \%$ for ZDV at concentrations of 6,500 and $800 \mathrm{ng} \mathrm{ml}^{-1}$, respectively. The mean inaccuracy of the assay ranged from $0.9 \%$ to $3.8 \%$ for $L M V$ and $-4.1 \%$ to $0.8 \%$ for ZDV over the calibration range. The calibration 
range for LMV and ZDV was $2-1000 \mathrm{ng} \mathrm{ml}^{-1}$ for both plasma and urine samples.

For EE and LN assays in study 3, plasma samples were analysed by Cephac Europe using previously validated methods. The methods for analysing EE and LN in plasma involved liquid-liquid extraction, purification by solid-phase extraction (C18 cartridge), followed by derivatization. The derivatives were analysed by gas chromatography/negative chemical ionization mass spectrometry. The overall imprecision coefficients of variation (CVs) for the analysis of plasma QC samples were $6.2 \%$, $9.3 \%$ and $5.8 \%$ for EE at target concentrations of 20, 200 and $450 \mathrm{pg} \mathrm{ml}^{-1}$, respectively. Mean inaccuracy of the assay ranged from $0.2 \%$ to $7.5 \%$ over the QC concentration range. The calibration range for EE was $10-500 \mathrm{pg} \mathrm{ml}^{-1}$. Overall method imprecision CVs for the analysis of plasma QC samples were $6.7 \%, 6.8 \%$ and $4.8 \%$ for $\mathrm{LN}$ at target concentrations of $0.3,10$ and $18 \mathrm{ng} \mathrm{ml}^{-1}$, respectively. Mean inaccuracy of the assay ranged from $0.0 \%$ to $4.4 \%$ over the QC concentration range. The calibration range for $L N$ was $0.1-20 \mathrm{ng} \mathrm{ml}^{-1}$. For maraviroc determination, plasma samples were analysed by Maxxam Analytics, Inc. using a validated method employing solid-phase extraction followed by liquid chromatography and mass spectrometric detection. The overall imprecision CVs for the analysis of QC samples were $4.0 \%, 1.8 \%$ and $2.9 \%$ for maraviroc at target concentrations of 1,90 and $180 \mathrm{ng} \mathrm{ml}^{-1}$, respectively. Mean inaccuracy of the assay ranged from -5.6 to $3.8 \%$ over the QC concentration range. The calibration range was $0.5-200 \mathrm{ng} \mathrm{ml}^{-1}$.

\section{Safety assessments}

Methods for assessment of safety were similar in the three studies described here. Information on adverse events (AEs) was collected throughout the study. AEs were considered as potentially related to study medication for 7 days after the final dose of maraviroc. All subjects underwent a urine drug screen to test for drugs of abuse at screening and at specified times during the studies. Subjects with a positive result did not enter into, or continue in, the studies. Physical examinations, supine and standing blood pressure/pulse rate measurements, 12 -lead electrocardiograms, and lab safety tests were conducted at appropriate intervals throughout each study.

\section{Statistical analysis}

In study 1 , it was estimated that a sample size of 12 subjects (six per sequence) would provide $90 \%$ confidence intervals (Cls) of \pm 0.164 and \pm 0.471 on the natural log scale for area under the plasma concentration-time curve (AUC) and maximum observed plasma concentration $\left(C_{\text {max }}\right)$, respectively, with $80 \%$ coverage probability. Assuming no change in AUC and $C_{\max }$, this relates to $90 \% \mathrm{Cls}$ of $84.9 \%$ to $118 \%$ and $62.4 \%$ to $160 \%$ for AUC and $C_{\max \text {, }}$ respectively. The standard deviation of differences (natural log scale) of 0.275 for AUC and 0.787 for $C_{\max }$ was calculated using estimates of variation from a previous study, for which the within-subject variances were 0.03775 for AUC and 0.30965 for $C_{\max }$ on the natural log scale.

In study 2, with a sample size of 12 subjects (six per sequence) and assuming no increase in AUC or $C_{\max }$, it was estimated that the $90 \% \mathrm{Cls}$ of the geometric mean ratios (GMRs) of maraviroc + LMV/ZDV compared with placebo + LMV/ZDV for LMV would be $92.6 \%$ to $108 \%$ for AUC and $82.6 \%$ to $121 \%$ for $C_{\max }$. Assuming a $100 \%$ increase in AUC or $C_{\max }$, it was estimated that the $90 \%$ Cls for LMV would be $185 \%$ to $216 \%$ for AUC and $165 \%$ to $242 \%$ for $C_{\text {max }}$. Similarly, assuming no increase in AUC or $C_{\max }$, it was estimated that the $90 \%$ Cls for ZDV would be $90.8 \%$ to $110 \%$ for AUC and $77.6 \%$ to $129 \%$ for $C_{\max }$. Assuming a $100 \%$ increase in AUC or $C_{\text {max }}$ it was estimated that the $90 \%$ Cls for ZDV would be $182 \%$ to $220 \%$ for AUC and $155 \%$ to $258 \%$ for $C_{\max }$. All calculations assumed a coverage probability of $80 \%$.

Given a sample size of 14 subjects (seven per sequence) in study 3 , and assuming no increase in AUC to the last measured concentration $\left(A \cup C_{t}\right)$ and $C_{\text {max }}$, it was estimated that the $90 \%$ Cls for EE would be $88.2 \%$ to $113 \%$ and $87.4 \%$ to $115 \%$, respectively, with $80 \%$ coverage probability. Assuming no increase in $A \cup C_{t}$ and $C_{\text {max }}$ the $90 \%$ Cls for $\mathrm{LN}$ would be $94.2 \%$ to $106 \%$ and $89.0 \%$ to $112 \%$, respectively, with $80 \%$ coverage probability.

Summary statistics for midazolam, LMV, ZDV, EE and LN pharmacokinetic parameters are presented by treatment. For each study, log transformed AUC, $A \cup C_{\tau}$ (where $\tau$ is the dosing interval) and/or $A \cup C_{t}$ (as appropriate to the study) and $C_{\max }$ and untransformed time to $C_{\max }\left(T_{\max }\right)$ for midazolam, LMV, ZDV, EE and LN were subjected to an analysis of variance including terms appropriate for each study design. Only subjects with data available for both treatment periods were included. The contrasts of interest were:

[maraviroc + midazolam (day 7)] vs. [placebo + midazolam (day 7)]

[maraviroc + LMV/ZDV (day 7)] vs. [placebo + LMV/ZDV (day 7)]

[maraviroc + EE/LN (day 8)] vs. [placebo $+E E / L N($ day 8)]

Statistical analyses were conducted using SAS software (SAS ${ }^{\circledR}$ Institute Inc., Cary, NC, USA; 1989, version 8.2).

\section{Results}

\section{Subjects}

All subjects in these studies were White. Subjects in study 1 ranged in age from 23 to 44 years (mean 31 years) and in weight from 55 to $91 \mathrm{~kg}$ (mean $70 \mathrm{~kg}$ ). Subjects in study 2 ranged in age from 21 to 45 years (mean 30 years) and in weight from 61 to $89 \mathrm{~kg}$ (mean $76 \mathrm{~kg}$ ). Subjects in study 3 ranged in age from 32 to 45 years (mean 38 years) and in weight from 57 to $76 \mathrm{~kg}$ (mean $65 \mathrm{~kg}$ ). In study 1, $12 \mathrm{sub}$ jects entered and completed the study (six per sequence) and were included in the pharmacokinetic and safety analyses. In study 2, 12 subjects entered the study and 11 


\section{Table 1}

Pharmacokinetics of midazolam in the presence and absence of maraviroc

\begin{tabular}{|c|c|c|c|c|c|}
\hline \multirow[b]{2}{*}{ Treatment } & \multicolumn{5}{|c|}{ Pharmacokinetic parameter } \\
\hline & $\begin{array}{l}\text { AUC } \\
\left(\text { (ng ml }^{-1} h\right)^{*} \\
\text { mean }(\mathrm{CV} \%)\end{array}$ & $\begin{array}{l}\text { AUC } \\
\left(\text { (ng ml-1 }^{-1}\right)^{*} \\
\text { mean }(\mathrm{CV} \%)\end{array}$ & $\begin{array}{l}C_{\max } \\
\left(\mathrm{ng} \mathrm{ml}^{-1}\right)^{*} \\
\text { mean }(\mathrm{CV} \%)\end{array}$ & $\begin{array}{l}T_{\max }(\mathrm{h}) \boldsymbol{\dagger} \\
\text { mean (SD) }\end{array}$ & $\begin{array}{l}t_{1 / 2}(h) \dagger \\
\text { mean (SD) }\end{array}$ \\
\hline Maraviroc + midazolam $(n=12)$ & $120(28)$ & $122(28)$ & $46.9(50)$ & $1.00(1.58)$ & $5.34(2.31)$ \\
\hline Placebo + midazolam $(n=12)$ & $102(33)$ & $104(33)$ & $38.7(44)$ & $0.79(0.45)$ & $5.25(1.51)$ \\
\hline Ratio (\%)‡ or difference§ & 118 & 118 & 121 & 0.21 & ND \\
\hline $90 \%$ confidence interval & 104,134 & 104,134 & $92.2,160$ & $-0.72,1.14$ & ND \\
\hline
\end{tabular}

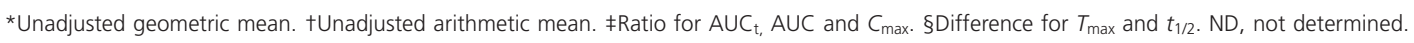

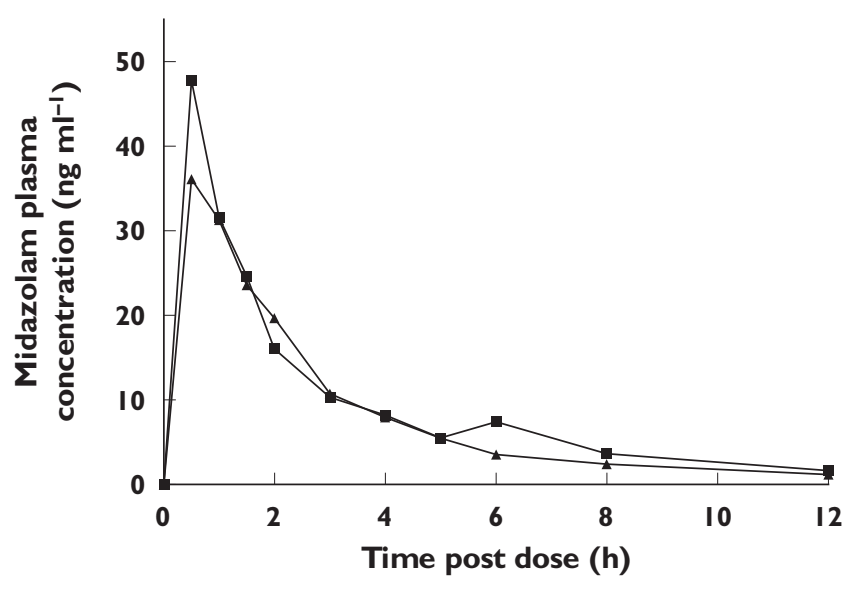

Figure 2

Plasma concentration profiles of midazolam in the presence and absence of maraviroc. Maraviroc + Midazolam ( $\mathbf{\square})$; Placebo + Midazolam ( $\mathbf{\Delta}$ )

completed the study. One subject discontinued during treatment with maraviroc+LMV/ZDV. Twelve subjects were included in the safety analysis and 11 in the pharmacokinetic analysis. In study 3, 15 subjects entered the study, and all subjects completed the study and were included in the analyses of pharmacokinetics and safety.

\section{Pharmacokinetics}

In study 1, the GMR of midazolam + maraviroc/ midazolam + placebo was $118 \%$ for both $A U C$ and $A U C_{t}$ $(90 \% \mathrm{Cl} 104,134)$ (Table 1, Figure 2). The GMR for $C_{\max }$ was $121 \%(90 \% \mathrm{Cl} 92.2,160)$. Midazolam $T_{\max }$ and terminal elimination half-life $\left(t_{1 / 2}\right)$ were similar when coadministered with maraviroc or placebo. Visual inspection of the maraviroc trough concentrations indicated that steady state was reached by day 7 (data not shown).

In study 2, the GMRs of LMV/ZDV + maraviroc vs. LMV/ ZDV + placebo were $114 \%(90 \% \mathrm{Cl} 98,132)$, for $\operatorname{LMV}\left(\mathrm{AUC}_{12}\right)$ and $98 \%(90 \% \mathrm{Cl} 79,122)$ for ZDV $\left(\mathrm{AUC}_{12}\right)$ (Table 2, Figure 3 ). Similarly, the GMRs for $C_{\max }$ were $116 \%(90 \% \mathrm{Cl} 87.5,154)$ for $L M V$ and $92 \%(90 \% \mathrm{Cl} 68,124)$ for $Z D V . T_{\max }$ and $\mathrm{CL}_{R}$ were

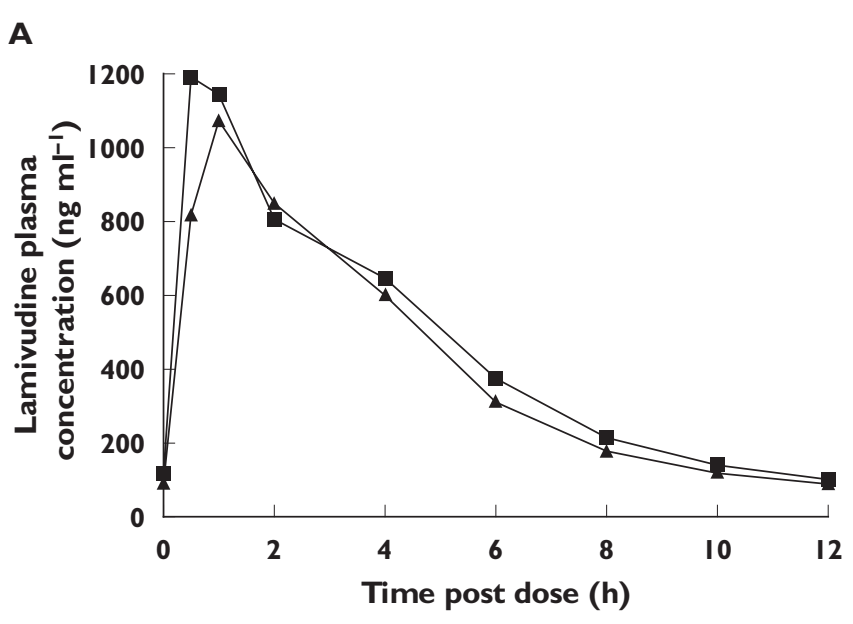

B

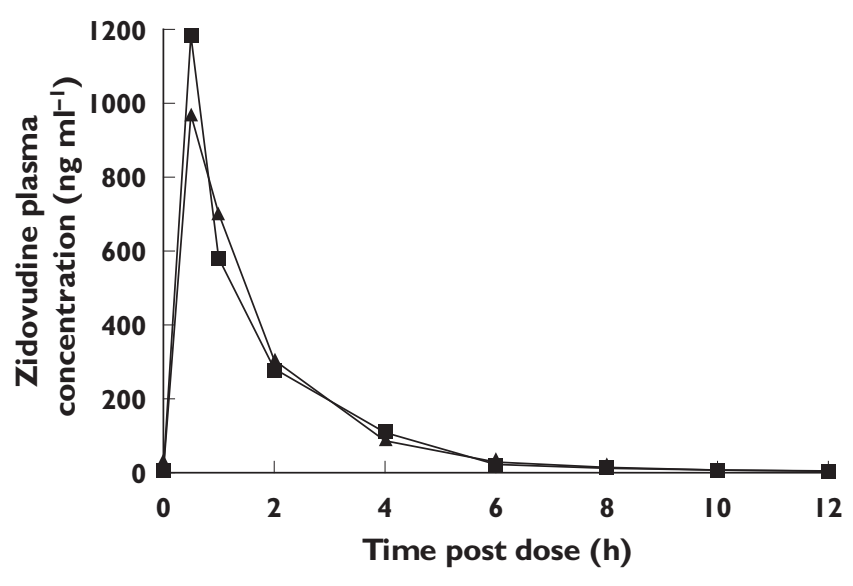

Figure 3

Plasma concentration profile of (A) lamivudine (LMV) and (B) zidovudine $(\mathrm{ZDV})$ in the presence and absence of maraviroc. Placebo + (A) LMV or (B) ZDV ( $\mathbf{\Delta}$ ); Maraviroc + LMV (A) or (B) ZDV ( $\mathbf{\square})$

similar for LMV and ZDV in the presence and absence of maraviroc.

In study 3, the GMR of EE/LN + maraviroc vs. EE/LN +placebo was $99.6 \%(90 \% \mathrm{Cl} 94.5,106)$ for $\mathrm{EE}\left(\mathrm{AUC} \mathrm{C}_{\mathrm{t}}\right)$ and 97.7\% $(90 \% \mathrm{Cl} 92,104)$ for $\mathrm{LN}\left(\mathrm{AUC}_{\tau}\right)$. The GMR and $90 \% \mathrm{Cls}$ 


\section{Table 2}

Pharmacokinetics of lamivudine (LMV) and zidovudine (ZDV) in the presence and absence of maraviroc

\begin{tabular}{|c|c|c|c|c|c|}
\hline \multicolumn{2}{|c|}{ Component } & \multicolumn{2}{|c|}{$\begin{array}{ll}\text { Pharmacokinetic parameter } & \\
\text { AUC }_{12} & \mathrm{C}_{\max } \\
\left(\mathrm{ng} \mathrm{ml}^{-1} \mathrm{~h}\right)^{*} & \left(\mathrm{ng} \mathrm{ml}^{-1}\right)^{*} \\
\text { mean (CV\%) } & \text { mean }(\mathrm{CV} \%)\end{array}$} & \multirow{2}{*}{$\begin{array}{l}\boldsymbol{T}_{\max }(\mathbf{h}) \boldsymbol{t} \\
\text { mean }(\mathrm{SD})\end{array}$} & \multirow{2}{*}{$\begin{array}{l}\mathrm{CL}_{R}\left(\mathbf{( ~ \mathbf { ~ } ^ { - 1 } ) \boldsymbol { \dagger }}\right. \\
\text { mean (SD) } \\
21.9(3.53)\end{array}$} \\
\hline \multirow[t]{4}{*}{ LMV } & Maraviroc + LMV/ZDV $(n=11)$ & $5491(15)$ & $1305(35)$ & & \\
\hline & Placebo + LMV/ZDV $(n=11)$ & $4852(24)$ & $1125(34)$ & $1.1(0.52)$ & $22.3(3.43)$ \\
\hline & Ratio (\%)‡ or difference§ & 114 & 116 & 0.1 & ND \\
\hline & $90 \%$ confidence interval & 98,132 & 88,154 & $-0.7,0.8$ & ND \\
\hline \multirow[t]{4}{*}{ ZDV } & Maraviroc + ZDV/LMV $(n=11)$ & $1685(36)$ & $1108(51)$ & $0.68(0.46)$ & $24.1(10.5)$ \\
\hline & Placebo + ZDV/LMV $(n=11)$ & $1713(36)$ & 1188 (39) & $0.68(0.25)$ & $21.4(5.7)$ \\
\hline & Ratio (\%)‡ or difference§ & 98 & 92 & 0.0 & ND \\
\hline & $90 \%$ confidence interval & 79,122 & 68,124 & $-0.3,0.3$ & ND \\
\hline
\end{tabular}

*Unadjusted geometric mean. +Unadjusted arithmetic mean. ¥Ratio for AUC 12 and $C_{\text {max. }}$ §Difference for $T_{\text {max. }}$ ND, not determined.

\section{Table 3}

Pharmacokinetics of ethinyloestradiol (EE) and levonorgestrel (LN) in the presence and absence of maraviroc

\begin{tabular}{|c|c|c|c|c|}
\hline \multicolumn{2}{|c|}{ Treatment } & $\begin{array}{l}\text { AUC }\left(\mathrm{pg} \mathrm{m}^{-1} \mathrm{~h}\right)^{*} \\
\text { mean }(\mathrm{CV} \%)\end{array}$ & $\begin{array}{l}C_{\max }\left(\mathrm{pg} \mathrm{ml}^{-1}\right)^{*} \\
\text { mean }(\mathrm{CV} \%)\end{array}$ & $\begin{array}{l}T_{\max }(\mathrm{h}) \dagger \\
\text { mean (SD) }\end{array}$ \\
\hline \multirow[t]{4}{*}{$\mathrm{EE}$} & Maraviroc + EE/LN & $745(30)$ & $84.0(28)$ & $1.20(0.53)$ \\
\hline & Placebo + EE/LN & $746(30)$ & $84.8(24)$ & $1.30(0.37)$ \\
\hline & Ratio $(\%) \neq$ or difference§ & 99.6 & 98.4 & -0.08 \\
\hline & $90 \%$ confidence interval & $94.5,105$ & $91.3,106$ & $-0.38,0.23$ \\
\hline \multirow[t]{4}{*}{ LN } & Maraviroc + EE/LN & $71(24)$ & $7.13(19)$ & $1.17(0.84)$ \\
\hline & Placebo + EE/LN & $72(28)$ & $7.05(22)$ & $0.97(0.40)$ \\
\hline & Ratio $(\%) \neq$ or difference & 97.7 & 100 & 0.21 \\
\hline & $90 \%$ confidence interval & 92,104 & 93,108 & $-0.21,0.62$ \\
\hline
\end{tabular}

*Unadjusted geometric means. +Unadjusted arithmetic means. $¥$ Ratio for $A U C_{12}$ and $C_{\text {max. }}$ §Difference for $T_{\text {max; }}$ AUC $C_{t}$ was calculated for EE instead of AUC $\tau$ because some subjects did not have measurable EE concentrations up to $24 \mathrm{~h}$ postdose.

were similar for both $C_{\max }$ and AUC for both compounds. $T_{\max }$ was similar for EE and LN in the presence and absence of maraviroc (Table 3, Figure 4). Visual inspection of maraviroc trough concentrations indicated that steady state was reached by day 6 (data not shown).

\section{Safety and tolerability}

Maraviroc was well tolerated in all studies, and no serious AEs were recorded. The majority of AEs were mild to moderate and transient in nature. The most common AEs were asthenia, headache and nausea, and one subject discontinued treatment with maraviroc and LMV/ZDV (study 2) due to AEs (severe flu syndrome, arthralgia and myalgia), some of which were considered treatment related. There were no dose reductions or temporary discontinuations during these studies.

\section{Discussion}

HIV is currently treated using multidrug regimens which target multiple, discrete stages in the virus life cycle. Fur- thermore, HIV-infected patients are susceptible to many comorbid illnesses, including a wide range of opportunistic infections, neoplasia [18], diabetes [19] and depression [20], which require additional complex drug regimens. In addition, many female patients are likely to be taking oral contraceptives. Therefore, it was important to investigate the potential for maraviroc to interact with co-administered therapeutics.

Maraviroc is extensively metabolized, primarily by CYP3A4 [6], with $\mathrm{CL}_{R}$ contributing a relatively minor portion (23\%) to total clearance of maraviroc [8]. Because CYP3A4 plays a role in the metabolism of many drugs [9], it is important to assess the potential of any new agent to affect the activity of this enzyme. In vitro and clinical data suggested that maraviroc is a substrate, but not an inhibitor or inducer of CYP3A4. The study conducted with midazolam, a probe CYP3A4 substrate which has been shown to be sensitive to modulators of CYP3A4 activity, confirmed that at clinically relevant doses, maraviroc had only a minor influence on the pharmacokinetics of midazolam, suggesting that there is a low potential for maraviroc to interfere with the metabolism of other drugs through this route. 
A

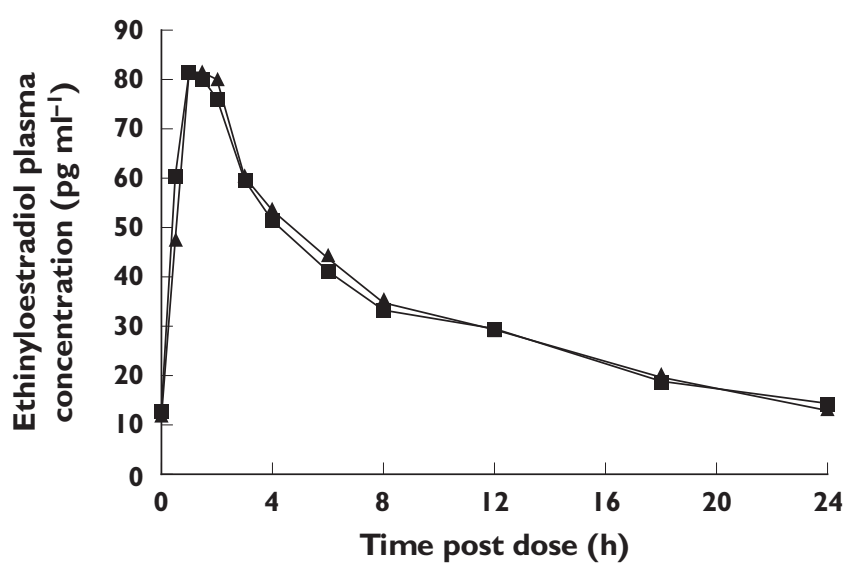

B

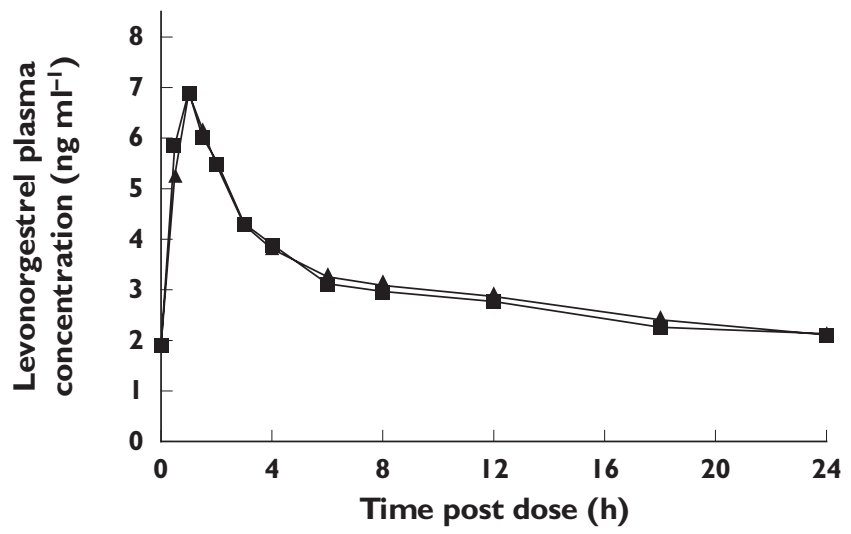

\section{Figure 4}

Plasma concentration profile of (A) ethinyloestradiol (EE) and (B) levonorgestrel $(\mathrm{LN})$ in the presence and absence of maraviroc. Maraviroc + (A) EE or (B) LN (ם); Placebo + (A) EE or (B) LN (A)

This is supported by findings that maraviroc at doses up to $600 \mathrm{mg}$ q.d. had no effect on urinary $6 \beta-\mathrm{OH}$ cortisol/ cortisol ratio in healthy volunteers, which suggests that maraviroc is not an inhibitor or inducer of CYP3A4 [7].

As maraviroc is a new drug for treating HIV infection, it will be used in combination with other antiretroviral drugs. The Phase $2 \mathrm{~b} / 3$ clinical programme in treatment-naive HIV patients involved co-administration of maraviroc with Combivir $^{\mathrm{TM}}$ (LMV and ZDV). Study 2 was conducted to assess the potential of maraviroc to affect the pharmacokinetics of LMV and ZDV, partly to underwrite co-administration in the advanced-phase clinical studies, and partly to investigate any potential for interaction with renal substrates. An analysis of the effects of maraviroc on the pharmacokinetics of LMV/ZDV showed no clinically relevant effect on the pharmacokinetics of LMV or ZDV. Co-administration of maraviroc $300 \mathrm{mg}$ b.i.d. with LMV/ ZDV BID for 7 days was well tolerated.

Women of childbearing age account for a large proportion of all HIV-infected patients, and many of them take oral contraceptives. Other drugs used in the treatment of HIV infection have been observed to reduce drug exposure of oral contraceptives $[21,22]$. It was therefore important to assess the potential influence of maraviroc on the pharmacokinetics of oral contraceptives as well as the safety of co-administering maraviroc with these compounds. This study has demonstrated that maraviroc had no effect on the pharmacokinetics of EE or $\mathrm{LN}$, and the combination of maraviroc $100 \mathrm{mg}$ b.i.d. $+\mathrm{EE} / \mathrm{LN}$ was well tolerated.

These studies suggest a low potential for interaction of maraviroc with other drugs that are either metabolized by CYP3A4 or renally cleared. Additionally, there is no clinically significant interaction with the oral contraceptive steroids EE and LN.

\section{Competing interests}

S.A., D.R., L.A.W., C.E.R. and G.J.M. were employed by Pfizer Ltd. at the time of this research.

These studies were sponsored by Pfizer. Editorial assistance was provided by Dylan Harris, PhD and Janet E. Matsuura, $\mathrm{PhD}$ at Complete Healthcare Communications, Inc, and was funded by Pfizer Inc, New York, NY, USA.

\section{REFERENCES}

1 Mousa O, Brater DC, Sunblad KJ, Hall SD. The interaction of diltiazem with simvastatin. Clin Pharmacol Ther 2000; 67: 267-74.

2 Olkkola KT, Backman JT, Neuvonen PJ. Midazolam should be avoided in patients receiving the systemic antimycotics ketoconazole or itraconazole. Clin Pharmacol Ther 1994; 55 : 481-5.

3 Karyekar CS, Eddington ND, Briglia A, Gubbins PO, Dowling TC. Renal interaction between itraconazole and cimetidine. J Clin Pharmacol 2004; 44: 919-27.

4 Yeni PG, Hammer SM, Hirsch MS, Saag MS, Schechter M, Carpenter CC, Fischl MA, Gatell JM, Gazzard BG, Jacobsen DM, Katzenstein DA, Montaner JS, Richman DD, Schooley RT, Thompson MA, Vella S, Volberdling PA. Treatment for adult HIV infection: 2004 recommendations of the International AIDS Society-USA Panel. JAMA 2004; 292: 251-65.

5 Tseng AL, Foisy MM. Management of drug interactions in patients with HIV. Ann Pharmacother 1997; 31: 1040-58.

6 Hyland R, Jones B, Muirhead G. In vitro assessment of the CYP-based drug-drug interaction potential of UK-427 857. In: 5th International Workshop on Clinical Pharmacology of HIV Therapy; 2004 April 1-3; Rome, Italy.

7 Abel S, van der Ryst E, Rosario MC, Ridgway CE, Medhurst CG, Taylor-Worth RJ, Muirhead GJ. Assessment of 
the pharmacokinetics, safety and tolerability of maraviroc, a novel CCR5 antagonist, in healthy volunteers. Br J Clin Pharmacol 2008; 65 (Suppl. 1): 5-18.

8 Abel S, Russell D, Whitlock LA, Ridgway CE, Nedderman ANR, Walker DK. Assessment of the absorption, metabolism, and absolute bioavailability of maraviroc in healthy male subjects. Br J Clin Pharmacol 2008; 65 (Suppl. 1): 60-67.

9 Zhou S, Chan E, Lim LY, Boelsterli UA, Li SC, Wang J, Zhang Q, Huang M, Xu A. Therapeutic drugs that behave as mechanism-based inhibitors of cytochrome P450 3A4. Curr Drug Metab 2004; 5: 415-42.

10 Galetin A, Ito K, Hallifax D, Houston JB. CYP3A4 substrate selection and substitution in the prediction of potential drug-drug interactions. J Pharmacol Exp Ther 2005; 314 : 180-90.

11 van Leeuwen R, Lange JM, Hussey EK, Donn KH, Hall ST, Harker AJ, Jonker P, Danner SA. The safety and pharmacokinetics of a reverse transcriptase inhibitor, 3TC, in patients with HIV infection: a phase I study. AIDS 1992; 6: 1471-5.

12 Singlas E, Pioger JC, Taburet AM, Colin JN, Fillastre JP. Zidovudine disposition in patients with severe renal impairment: influence of hemodialysis. Clin Pharmacol Ther 1989; 46: 190-7.

13 Moore KH, Yuen GJ, Raasch RH, Eron JJ, Martin D, Mydlow PK, Hussey EK. Pharmacokinetics of lamivudine administered alone and with trimethoprim-sulfamethoxazole. Clin Pharmacol Ther 1996; 59: 550-8.

14 de Miranda P, Good SS, Yarchoan R, Thomas RV, Blum MR, Myers $\mathrm{CE}$, Broder $\mathrm{S}$. Alteration of zidovudine pharmacokinetics by probenecid in patients with AIDS or AIDS-related complex. Clin Pharmacol Ther 1989; 46: 494-500.

15 Ebner T, Remmel RP, Burchell B. Human bilirubin UDP-glucuronosyltransferase catalyzes the glucuronidation of ethinylestradiol. Mol Pharmacol 1993; 43:649-54.

16 Wang B, Sanchez RI, Franklin RB, Evans DC, Huskey SE. The involvement of CYP3A4 and CYP2C9 in the metabolism of 17 alpha-ethinylestradiol. Drug Metab Dispos 2004; 32: 1209-12.

17 Yasuda S, Suiko M, Liu MC. Oral contraceptives as substrates and inhibitors for human cytosolic SULTs. J Biochem (Tokyo) 2005; 137: 401-6.

18 Bower M, Palmieri C, Dhillon T. AIDS-related malignancies: changing epidemiology and the impact of highly active antiretroviral therapy. Curr Opin Infect Dis 2006; 19: 14-9.

19 Hadigan C. Diabetes, insulin resistance, and HIV. Curr Infect Dis Rep 2006; 8: 69-75.

20 Valente SM. Depression and HIV disease. J Assoc Nurses AIDS Care 2003; 14: 41-51.

21 Ouellet D, Hsu A, Qian J, Locke CS, Eason CJ, Cavanaugh JH, Leonard JM, Granneman GR. Effect of ritonavir on the pharmacokinetics of ethinyl oestradiol in healthy female volunteers. Br J Clin Pharmacol 1998; 46: 111-6.

22 Back D, Gibbons S, Khoo S. Pharmacokinetic drug interactions with nevirapine. J Acquir Immune Defic Syndr 2003; 34 (Suppl. 1): S8-14. 